Enferm Bras 2020;19(5):403-10

https://doi.org/10.33233/eb.v19i5.4086

\title{
ARTIGO ORIGINAL \\ Sintomatologia depressiva em enfermeiros que atuam na sala vermelha de um hospital público do município de Caruaru/PE
}

Fernanda Caroline Florêncio*, Geraldo Vicente Nunes Neto**, Ana Maria Sá Barreto Maciel, M.Sc. ${ }^{* *}$, Gidelson Gabriel Gomes, M.Sc. ${ }^{* * *}$, Yalle Laryssa Florencio Silva ${ }^{* * * *}$, Edson Dias Barbosa Neto ${ }^{* \star * * *}$

*Enfermeira, graduada pelo Centro Universitário Tabosa de Almeida ASCES-UNITA, Especializando na modalidade residência em ortopedia e traumatologia no Hospital Getúlio Vargas, ${ }^{* *}$ Enfermeiro, graduado pelo Centro Universitário Tabosa de Almeida ASCES-UNITA, residência em terapia intensiva do Real Hospital Português (UFPE), ${ }^{* * *}$ Docente do curso de Enfermagem do centro Universitário Tabosa de Almeida ASCES-UNITA, ${ }^{* * * * E n f e r m e i r o, ~}$ Docente do curso de enfermagem do Centro Universitário do Vale do Ipojuca UNIFAVIP/Wyden, Caruaru/PE, Gestor acadêmico e professor do Gabaritando Carreiras, Supervisor de Enfermagem no Hospital Regional de Agreste (HRA), Preceptor do programa de residência de enfermagem em UTI geral do Hospital Regional do Agreste, Especialista em Auditoria em Serviços de Saúde - Faculdade Redentor - IDE Cursos, ${ }^{* * * * * E n f e r m e i r a, ~ g r a d u a d a ~ p e l o ~ C e n t r o ~}$ Universitário Tabosa de Almeida ASCES-UNITA, Especialização em Saúde Pública com ênfase em saúde da família (INESP), Especialização em Saúde mental, crack, álcool e outras drogas (INESP), ${ }^{* * * * *}$ Graduado em Enfermagem pela Universidade Estadual do Pernambuco (UPE), Especialista em neurologia, residente em Terapia intensiva RHP

Recebido em 3 de maio de 2020; aceito em 23 de outubro de 2020.

Correspondência: Ana Maria Barreto Sá Maciel, Rua Pastor Rubem Prado, 50/201, 55014-395 Caruaru PE

Ana Maria Barreto Sá Maciel: anabarreto@asces.edu.br

Fernanda Caroline Florêncio: nanyf0110@gmail.com

Geraldo Vicente Nunes Neto: geraldonunes.enf@gmail.com

Gidelson Gabriel Gomes: gidelsongabrielgomes@gmail.com

Yalle Laryssa Florencio Silva: yalleflorencio@gmail.com

Edson Dias Barbosa Neto: edson.utirhp@gmail.com

\section{Resumo}

Objetivo: Verificar a presença de sintomatologia depressiva em enfermeiros que trabalham na sala vermelha. Métodos: Trata-se de um estudo descritivo, transversal com abordagem quantitativa no qual enfermeiros que atuam na sala vermelha foram abordados a fim de detectar a sintomatologia depressiva por meio da Escala de depressão de Beck. Resultados: Dos enfermeiros entrevistados, $64,29 \%$ apresentam grau mínimo de sintomas depressivos, $28,57 \%$ demonstram sintomas de leve a moderado e $7,14 \%$ demonstrou sintomas moderado a grave de acordo com escores do BDI estabelecidos para este estudo. Conclusão: Por meio do presente estudo foi possível observar o acometimento da sintomatologia depressiva nos enfermeiros da sala vermelha, como também a gama de fatores que estão relacionadas com o surgimento da depressão ou até mesmo a síndrome de Burnout.

Palavras-chave: depressão, enfermagem, emergências.

\section{Abstract \\ Depressive symptomatology in nurses who work in the red room of a public hospital in the municipality of Caruaru/PE}

Objective: To verify the presence of depressive symptoms in nurses who work in the red room. Methods: This is a descriptive, cross-sectional study with a quantitative approach in which nurses working in the red room were approached in order to detect depressive symptoms using the Beck Depression Inventory Scale. Results: Of the nurses interviewed, $64.29 \%$ had a minimum degree of depressive symptoms, $28.57 \%$ showed mild to moderate symptoms and $7.14 \%$ showed moderate to severe symptoms according to BDI scores established for this study. Conclusion: 
Through this study, it was possible to observe the impairment of depressive symptoms in nurses in the red room, as well as the range of factors that are related to the onset of depression or even Burnout syndrome.

Keywords: depression, nursing, emergencies.

\section{Resumen \\ Sintomatología depresiva en enfermeros que actúan en la sala roja de un hospital público del municipio de Caruaru/PE}

Objetivo: Verificar la presencia de síntomas depresivos en enfermeras que trabajan en la sala roja. Métodos: Este es un estudio descriptivo, de corte transversal con un enfoque cuantitativo en el que se abordó a los enfermeros que trabajan en la sala roja para detectar síntomas depresivos utilizando la escala del Inventario de Depresión de Beck (BDI). Resultados: De las enfermeras entrevistadas, el $64.29 \%$ tenía un grado mínimo de síntomas depresivos, el $28.57 \%$ mostró síntomas leves a moderados y el $7.14 \%$ mostró síntomas moderados a severos de acuerdo con los puntajes de BDI establecidos para este estudio. Conclusión: A través de este estudio, fue posible observar el deterioro de los síntomas depresivos en los enfermeros en la sala roja, así como el rango de factores que están relacionados con el inicio de la depresión o incluso el síndrome de Burnout.

Palabras-clave: depresión, enfermería, urgencias médicas.

Introdução

A depressão é caracterizada como um estado de sofrimento psíquico que tem resultado sobre as relações interpessoais, é identificada pela alteração do humor e pelo prolongamento de sintomas depressivos. Ela é um fator de risco para enfermidades, pois a queixa clínica da patologia pode ser mascarada pela sintomatologia deste transtorno [1]. A pessoa acometida desse transtorno de humor pode queixar-se de cefaleia persistente, dispepsia, falta de apetite, constipação, e por apresentar indisposição, indecisão e insegurança, pode comprometer sua rotina de trabalho, sua satisfação pessoal e saúde [2].

Por apresentar alta incapacidade laborativa, alta morbidade e mortalidade a depressão é considerada um problema de saúde pública. O indivíduo pode apresentar desânimo, pessimismo, podendo causar também diminuição da autoestima e do interesse social e sexual, insônia, falta de apetite, sentimentos autopunitivos e descrença em capacidades individuais [2].

Somado a isto, a depressão enquanto condição de sofrimento psíquico influencia na longevidade e qualidade de vida, podendo atingir níveis de incapacidade e invalidez. Ao relatar a associação do trabalho como evento estressor e de sofrimento psíquico, a classe trabalhista dos profissionais de enfermagem merece atenção e pesquisas que busquem entender e amenizar eventos que levem ao comprometimento psicossocial do trabalhador [3].

Por comprometer a rotina de trabalho, a satisfação pessoal e saúde dos profissionais, os profissionais de enfermagem em especial merecem atenção no tocante a sintomatologia depressiva, pois se enquadram no grupo de profissionais da área da saúde mais propensos aos problemas de saúde mental, o que toma amplitude relevante e está entre as três doenças psíquicas mais prevalentes nessa classe trabalhista [1].

A sala vermelha é preparada para receber pacientes classificados como urgência absoluta, que se encontram em situação crítica, com quadro clínico instável e risco iminente de morte, em diferentes faixas etárias, necessitando de intervenções médicas clínicas ou cirúrgicas [4]. Muitas vezes, a sobrecarga de trabalho e as diversas funções que o enfermeiro desempenha neste setor pode desencadear fadiga física e emocional, fatores estes que predispõem ao aparecimento de sintomas depressivos [5].

O enfermeiro da sala vermelha tem uma rotina de trabalho avaliada como desencadeadora de desgaste físico, emocional e de estresse, pois o ambiente ao qual está inserido exige condutas tão rápidas que, em alguns momentos, demandam ações simultâneas sem prévios planejamentos, sendo responsável pelo bem-estar e vida dos pacientes, que possuem risco iminente de morte e complexidade na prestação de cuidados, sendo então situações desencadeadoras do estresse ocupacional [3].

Desta forma, discutir possíveis relações entre trabalho e saúde mental dos enfermeiros da sala vermelha apresentou-se relevante, visto que a ideia de contemplar essas possíveis relações parece bastante atraente, por este ser um trabalhador do campo da saúde, que tem nas suas atribuições lidar com o manejo das dores e fragilidades humanas. 
Diante do exposto, este trabalho teve como objetivo verificar a presença de sintomatologia depressiva em enfermeiros que trabalham na sala vermelha, visto que estes profissionais lidam com uma rotina considerada estressante e exaustiva, devido à sobrecarga $e$ inadequação das condições de trabalho, somada ao desgaste físico e emocional oriundos do setor como revelam outros estudos literários.

Material e métodos

Trata-se de um estudo descritivo, transversal com abordagem quantitativa no qual enfermeiros que atuam na sala vermelha foram abordados a fim de detectar a sintomatologia depressiva por meio da Escala de Depressão de Beck (Beck Depression Inventory). Foram incluídos no estudo enfermeiros assistenciais que atuam na sala vermelha há mais de 6 meses. Considerou-se como critério de exclusão: enfermeiros que trabalham na sala vermelha há no mínimo 6 meses, pois os profissionais que estão em um tempo inferior a esse podem não expressar ainda o perfil de profissionais do setor e enfermeiros que estavam afastados de suas atividades no setor, seja por férias, atestado ou licença.

O estudo respeitou todos os aspectos éticos em pesquisa, de acordo com as Resoluções 466/12 e 510/16 do Conselho Nacional de Saúde (CNS), visando à preservação da autonomia, não-maleficência, beneficência e justiça. Foi submetido à Plataforma Brasil e aprovado pelo Comitê de Ética em Pesquisa da Associação Caruaruense de ensino superior e técnico (ASCES) sob o protocolo CAAE - 90294618.4.0000.5203.

A amostra foi do tipo censitária, em virtude de haver uma pequena quantidade de enfermeiros no setor em tela, não havendo, portanto, necessidade de cálculo, perfazendo, portanto, um total de 14 participantes. O estudo foi realizado no setor da sala vermelha de um hospital estadual situado no município de Caruaru/PE, tal serviço é referência na macrorregional Caruaru para o atendimento de emergência em traumato-ortoperdia, cirurgia geral e bucomaxilo-facial de alta complexidade [6].

As entrevistas ocorreram no período de dezembro de 2018 a fevereiro de 2019. Para apreensão de informações sociodemográficas foi utilizado um questionário semiestruturado, autoaplicável construído pelos autores, e para obtenção dos dados relativos à sintomatologia depressiva foi utilizado um instrumento estruturado, nomeado por: Escala de depressão de Beck Beck Depression Inventory (BDI), que foi criada por Aaron Beck, e consiste em um instrumento composto por 21 itens de múltipla escolha, e com itens relacionados a sintomatologias depressivas como: tristeza, desânimo, sensação de fracasso, sentimentos de estar sendo punido, decepção, ideação suicida, irritabilidade, perda de interesse e energia, padrão de sono e alimentar comprometido e diminuição da libido. Por tratar-se de um questionário autoaplicável serão eliminados aqueles que não forem respondidos integralmente [7].

A soma total da pontuação dessas questões apresenta um escore mínimo e máximo de zero a 63. Esse escore mostra o grau de severidade da depressão, que são classificados como mínima ou sem depressão (<10 pontos); leve a moderada (10-18 pontos); moderada a grave (1929 pontos); grave (30-63 pontos) [7].

Os dados foram digitados no Excel da Microsoft em seguida transferidos para o software estatístico Epiinfo versão 7.2, onde foram processados e analisados. A estatística utilizada foi do tipo descritiva por distribuição de frequência absolutas e relativas, sendo os resultados apresentados por meio de gráficos e tabelas.

Resultados

\section{Características da amostra}

A amostra obtida constitui-se de 14 enfermeiros com idade média de 33,43 anos. De acordo com os dados sociodemográficos, $57,1 \%$ dos enfermeiros são casados, $35,7 \%$ são solteiros e apenas $7,1 \%$ é viúvo. Em relação a ter filhos, $50 \%$ responderam que têm filhos e os outros 50 \% não tem filhos. No que concerne ao tempo de formação, todos são formados há mais de 1 ano, dos quais $42,7 \%$ são formados há mais de 10 anos. Desses, 35,7\% apenas trabalham e $64,3 \%$ trabalham e estudam. No que diz respeito ao grau de instrução, $21,4 \%$ possui à graduação, $71,4 \%$ está cursando ou concluiu a pós-graduação e apenas $7,1 \%$ possui mestrado.

Dos 14 respondentes, todos trabalham na sala vermelha do HRA há mais de 6 meses, $42,8 \%$ trabalham nesse setor há mais de 2 anos e 14,2\% há mais de 10 anos. Ao ser indagados 
se desejam mudar de setor, apenas $7,1 \%$ dos entrevistados manifestou o desejo, e os demais $92,9 \%$ não querem mudar de setor.

Tabela I - Distribuição dos dados sociodemográficos dos participantes da pesquisa, Caruaru/PE, 2019.

\begin{tabular}{lll} 
& Media (anos) & $\%$ \\
\hline Idade & 33,43 & \\
Estado civil & & 57,14 \\
Solteiro & & 35,72 \\
Casadol União estável & 7,14 \\
Viúvo (a) & \\
\hline Filhos & 60 \\
Sim & & 40 \\
Não & 9,07 & \\
Tempo de formação & 4,14 & \\
Tempo que trabalha na sala vermel ha & \\
Grau de instrução & & 21,43 \\
Graduação & & 71,43 \\
Pós-graduação & & 7,14 \\
Mestrado & \\
Atividades desenvolvida atualmente & & 64,3 \\
Trabalha e estuda & 35,7 \\
Apenas trabalha & & \\
Desejo de mudar de setor & & 7,1 \\
Sim & & 92,9 \\
Não & \\
\hline
\end{tabular}

Fonte: Autores, 2019.

Dos 21 itens avaliados pelo inventário de Beck, aplicado no estudo, algumas variáveis se mostraram importantes e promissores frente à avaliação da depressão em enfermeiros que atuam na sala vermelha. A tabela II ilustra a avaliação dos itens supracitados:

Tabela II - Inventário de Beck, Caruaru/PE, 2019. (ver anexo em PDF)

Prevalência de depressão, segundo o IDB

Dos enfermeiros entrevistados, 64,29\% apresentam grau mínimo de sintomas depressivos, $28,57 \%$ demonstram sintomas de leve a moderado (disforia) e $7,14 \%$ demonstraram sintomas moderado a grave de acordo com escores do BDI estabelecidos para este estudo. (Tabela III).

Tabela III - Distribuição dos participantes de acordo com a prevalência de depressão, segundo o Beck Depression Inventory (BDI).

\begin{tabular}{lll}
\hline Grau & $\mathbf{N}^{\circ}$ & $\%$ \\
\hline Mínimo (0-9) & 9 & $64,29 \%$ \\
Leve a moderada (10-18 pontos) & 4 & $28,57 \%$ \\
Moderada a grave - disforia (19-29 pontos) & 1 & $7,14 \%$ \\
Grave (30-63 pontos) & 0 & $0 \%$ \\
Total & 14 & $100 \%$ \\
\hline
\end{tabular}

Fonte: Autores, 2019.

Discussão

O enfermeiro, que atua na sala vermelha é aquele que presta cuidados a indivíduos de todas as idades, com alterações da saúde física e psíquica que necessitam de intervenções médicas clínicas ou cirúrgicas. Muitas vezes, a sobrecarga de trabalho e as diversas funções que o enfermeiro desempenha podem desencadear fadiga física e emocional, tensão, ansiedade no profissional [5]. Com base nessas evidências, aplicou-se o IDB, instrumento de autoavaliação, amplamente reconhecido em diversos países, que avalia a prevalência, a sintomatologia depressiva e intensidade de depressão. 


\section{Associação da depressão com os dados sociodemográficos}

Quanto ao estado civil, $57,1 \%$ dos enfermeiros são casados, $35,7 \%$ são solteiros e apenas $7,1 \%$ é viúvo. De acordo com a literatura, o predomínio da sintomatologia depressiva é maior nos enfermeiros separados ou divorciados, porém, segundo estudos da literatura nacional e internacional, comprovou-se ligação entre a presença da sintomatologia depressiva e o estado civil [8]. Enfermeiros divorciados ou separados apresentaram maiores taxas de depressão do que os solteiros e casados. Frente a esse resultado, infere-se que uma base familiar e o cônjuge tem relação inversa com o desenvolvimento de estresse e doenças físicas e da depressão e que, em indivíduos sem esse tipo de base, há elevação nas taxas de estresse ocupacional [9].

Observou-se também que ter filho apresentou associação significativa com os sintomas preditores da depressão em mulheres, resultado contraditório ao encontrado no estudo em questão, que mostrou uma maior prevalência da sintomatologia depressiva nos profissionais que não tem filhos. Os profissionais da enfermagem com filhos experimentam menos sintomas depressivos do que os sem filhos devido ao suporte familiar e ao trinômio (mãe, filho e pai) [1].

Os enfermeiros com duplas atividades (trabalham e estudam) apresentam uma maior prevalência da sintomatologia depressiva. De acordo com os resultados, $35,7 \%$ apenas trabalham e 64,3\% trabalham e estudam [1]. A literatura comprova associação significativa dessa variável com a sintomatologia depressiva [10]. De acordo com um estudo realizado em hospitais do noroeste do estado de São Paulo, profissionais de enfermagem que trabalhavam e estudavam jornada dupla tinham 2 a 11 vezes a chance de apresentarem a sintomatologia depressiva em relação aos enfermeiros que apenas trabalhavam. Estar engajado em duplas atividades pode ser associada à sintomatologia depressiva nestes trabalhadores devido a privação do lazer e convívio social necessários a saúde mental $[9,10]$.

\section{Associação da depressão com o BDI}

Insônia, inatividade física e insatisfação com padrão do sono foram aspectos fortemente associados à prevalência de depressão entre os profissionais da sala vermelha. $57,1 \%$ dos entrevistados não consegue conciliar bem o sono e repouso; $71,4 \%$ ficam cansados com mais facilidade e $50 \%$ estão preocupados com problemas físicos, dores e aflições. Esses fatores estão diretamente associados com a sintomatologia depressiva, que é caracterizada por tristeza ou irritabilidade, desinteresse ou desprazer, sentimento de culpa ou baixa autoestima, distúrbios do sono ou apetite, fadiga, entre outros [11]. Não resta dúvida de que a quantidade inadequada de sono e repouso pode diminuir o bem-estar pela execução de atividades do dia a dia e pode trazer outros problemas de saúde, podendo ser a ansiedade, a síndrome de burnout ou até mesmo mialgia uma condição presente nessas situações [12].

Cerca de $57,1 \%$ dos entrevistados não consegue dormir tão bem como costumava; $71,4 \%$ se sente exausto (fica cansado com mais facilidade) e 7,1\% sente-se cansado para realizar qualquer atividade. Existiu uma correlação em que quanto maior foi o nível de esgotamento emocional e de desrealização (despersonalização), maior foi a sintomatologia depressiva; e quanto menor a realização profissional (inversamente proporcional), maior a sintomatologia depressiva [10].

O aumento do número de casos da síndrome de burnout e da sintomatologia depressiva são preocupantes. Foi observado também que a Política Nacional de Saúde do Trabalhador encontra-se distante de suprir as necessidades dos profissionais que atuam na sala vermelha. É primordial que sejam adotadas ferramentas de enfrentamento contra o burnout e a sintomatologia depressiva no contexto ocupacional, uma vez que isso pode refletir em prejuízos na qualidade da assistência prestada a pacientes intensivos e semi-intensivos, assim como na taxa de absenteísmo [14].

\section{Conclusão}

Por meio do presente estudo foi possível observar o acometimento da sintomatologia depressiva nos enfermeiros da sala vermelha, como também a gama de fatores que está relacionada com o surgimento da depressão ou até mesmo a síndrome de burnout. 
A presença da sintomatologia depressiva era baixa na população estudada, mas os resultados despertam preocupação, uma vez que algum dos seus sentimentos, como ansiedade, tristeza, pessimismo, sensação de punição, irritabilidade, sensação de choro constante, retração social e algumas atitudes constituintes podem estar presente de modo intermitente, avançando com 0 tempo. A sobrecarga de trabalho, dificuldades na escala, execução de tarefas, atendimento ao paciente, o esgotamento, o suporte social entre outros aspectos, consubstanciados nos níveis altos e médios de exaustão emocional levando a esses profissionais a predispor sintomas depressivos comprometimento da qualidade de vida no trabalho.

As limitações de informações relativas sobre a temática representam desafios que devem ser superados com pesquisas futuras, pois é de grande valia e de relevância para a área da saúde mental e de suma importância para avaliar a qualidade de vida dos profissionais de enfermagem, bem como o aperfeiçoamento da política que vincula a saúde do trabalhador e os impactos que isso produz na vida desses profissionais.

\section{Referências}

1. Vasconcelos EM, Martino MMF. Preditores da sintomatologia depressiva em enfermeiros de unidade de terapia intensiva. Esc Anna Nery 2017;21(3):e20170031. https://doi.org/10.1590/2177-9465-ean-2017-0031

2. Marconi EVN. Depressão infantil: uma revisão bibliográfica. Psicologia.pt - O Portal dos psicólogos; 2017. [citado 2020 Feb 10]. Available from: https://www.psicologia.pt/artigos/textos/A1091.pdf

3. Oliveira FP, Mazzaia MC, Marcolan JF. Sintomas de depressão e fatores intervenientes entre enfermeiros de serviço hospitalar de emergência. Acta Paul Enferm 2015;28(3):209-15. https://doi.org/10.1590/1982-0194201500036

4. Silva PL, Paiva L, Faria VB, OhI RIB, Chavaglia SRR. Acolhimento com classificação de risco do serviço de Pronto-Socorro Adulto: satisfação do usuário. Rev Esc Enferm USP 2016;50(3):427-33. https://doi.org/10.1590/S0080-623420160000400008

5. Santos DR. O papel do enfermeiro no atendimento emergencial ao paciente vítima de infarto agudo do miocárdio na sala vermelha: uma revisão e literatura. Revista de Ciências Humanas (RCH) 2014. [citado2020 Feb 10]. Available from: https://repositorio.ufsc.br/xmlui/handle/123456789/173411

6. Brasil. Pernambuco. Secretaria Estadual de Saúde. Hospital Regional do Agreste Dr. Waldemiro Ferreira. [citado 2020 Feb 10]. Available from:

http://portal.saude.pe.gov.br/unidades-de-saude-e-servicos/secretaria-executiva-deatencao-saude/hospital-regional-do-agreste-dr

7. Oliveira MHG, Gorenstein C, Lotufo Neto F, Andrade LH, Wang YP. Validation of the Brazilian Portuguese version of the Beck Depression Inventory-II in a community sample. Rev Bras Psiquiatr 2012;34(4):389-94.

https://doi.org/10.1016/j.rbp.2012.03.005

8. Galindo RH, Oliveira FKV, Lima RAS, Souza Al. Síndrome de Burnout entre enfermeiros de um hospital geral da cidade do Recife. Rev Esc Enferm USP 2012;46(2):420-7. https://doi.org/10.1590/S0080-62342012000200021

9. Vargas D, Dias APV. Prevalência de depressão em trabalhadores de enfermagem de Unidade de Terapia Intensiva: estudo em hospitais de uma cidade do noroeste do Estado São Paulo. Rev Latinoam Enferm 2011;19(5):1114-21.

https://doi.org/10.1590/S0104-11692011000500008

10. Perry L, Lamont S, Brunero S, Gallagher R, Duffield $C$. The mental health of nurses in acute teaching hospital settings: a cross-sectional survey. BMC Nurs 2015;14:18. https://doi.org/10.1186/s12912-015-0068-8

11. Leão AM, Gomes IP, Ferreira MJM, Cavalcanti LPG. Prevalência e fatores associados à depressão e ansiedade entre estudantes universitários da área da saúde de um Grande Centro Urbano do Nordeste do Brasil. Rev Bras Educ Med 2018;42(4):55-65. https://doi.org/10.1590/1981-52712015v42n4rb20180092

12. Galvão A, Pinheiro M, Gomes MJ, Ala S. Ansiedade, stress e depressão relacionados com perturbações do sono-vigília e consumo de álcool. Revista Portuguesa de Enfermagem de Saúde Mental 2017;(spe5):8-12. https://doi.org/10.19131/rpesm.0160

13. Vasconcelos EM, Martino MMF, França SPS. Burnout e sintomatologia depressiva em enfermeiros de terapia intensiva: análise de relação. Rev Bras Enferm 2018;71(1):13541. https://doi.org/10.1590/0034-7167-2016-0019 